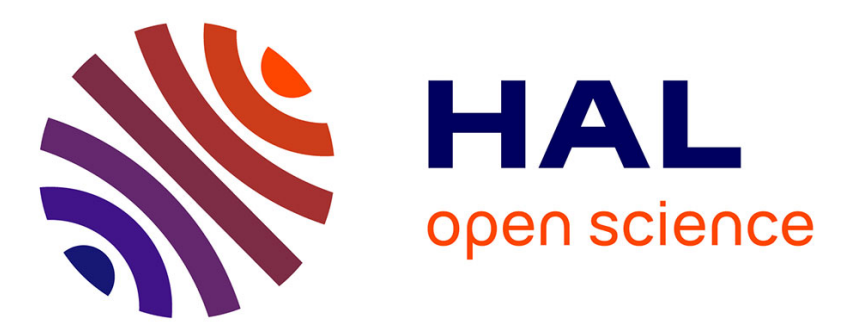

\title{
Segmented wavefront metrology using multicolor PISTIL interferometry
}

\author{
Bastien Rouzé, Cindy Bellanger, Patrick Lanzoni, Frederic Zamkotsian,
} Jerôme Primot

\section{- To cite this version:}

Bastien Rouzé, Cindy Bellanger, Patrick Lanzoni, Frederic Zamkotsian, Jerôme Primot. Segmented wavefront metrology using multicolor PISTIL interferometry. SPIE Photonics Europe 2020, Mar 2020, STRASBOURG, France. 10.1117/12.2553084 • hal-02932094

\section{HAL Id: hal-02932094 \\ https://hal.science/hal-02932094}

Submitted on 7 Sep 2020

HAL is a multi-disciplinary open access archive for the deposit and dissemination of scientific research documents, whether they are published or not. The documents may come from teaching and research institutions in France or abroad, or from public or private research centers.
L'archive ouverte pluridisciplinaire HAL, est destinée au dépôt et à la diffusion de documents scientifiques de niveau recherche, publiés ou non, émanant des établissements d'enseignement et de recherche français ou étrangers, des laboratoires publics ou privés. 


\title{
Segmented wavefront metrology using multicolor PISTIL interferometry
}

\author{
Bastien Rouzéa ${ }^{\mathrm{a}}$, Cindy Bellanger ${ }^{\mathrm{a}}$, Patrick Lanzoni ${ }^{\mathrm{b}}$, \\ Frédéric Zamkotsian ${ }^{\mathrm{b}}$, and Jérome Primot ${ }^{\mathrm{a}}$

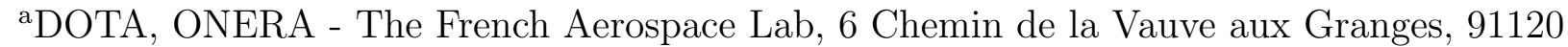 \\ Palaiseau, France \\ b Aix Marseille Université, CNRS, CNES, LAM - Laboratoire d'astrophysique de Marseille, 38 \\ Rue Frédéric Joliot Curie, 13013 Marseille, France
}

\begin{abstract}
PISTIL (Piston and Tilt) is a recent interferometric system that computes the absolute piston and tip/tilt map of a segmented wavefront. Its high precision makes it usable as a metrology tool for wavefront sensing of coherently-combined laser arrays for example. This interferometer needs to correctly address high dynamic piston sensing, while dealing with fringes wrapping that leads to ambiguous phase estimations. We derived a mathematical combination for two measurements at different wavelengths and did a technical demonstration of it, using a IRIS-AO PTT111 Deformable Mirror as a segmented wavefront generator. We have verified that the loss of accuracy is slightly increased for a larger piston compared to a previous study, and we got a standard error of $\lambda / 160$ with a Peak-to-valley of $\lambda / 50$. This technique could be extended to a broader spectrum.
\end{abstract}

Keywords: Segmented wavefront, Wavefront analysis, Two-wavelength interferometry, PISTIL interferometry

\section{INTRODUCTION}

Today, segmented wavefronts appear in various fields of Optics (Figure 1), notably in the domain of new highpower lasers. Indeed, these designs rely largely on the coherent combination of elementary sources, ${ }^{1-5}$ which are often collimated amplified fiber lasers. When they propagate into the far field, they may interfere constructively (increasing the output power) whenever all the lasers have the same phase, i.e. the segmented wavefront, generated by the union of each subwavefront from the collimated beams, is flattened. The design of these highpower lasers is thus based on the design of a control-command loop for the segmented wavefront shaping of the elementary beams, either in pupil plane (heterodyne detection and lateral-shearing interferometry) ${ }^{6-8}$ or in far field (LOCSET, SPGD). ${ }^{9,10}$ On another hand, in astronomy, the primary mirror of the new giant telescopes will be made of a few (like GMT) ${ }^{11}$ to hundreds (like ELT) ${ }^{12}$ smaller pieces. For these segmented telescopes, a co-phasing step is necessary prior to performing observations. New cophasing means have been developed, such as broadband phasing, ${ }^{13}$ and phase masks. ${ }^{14,15}$

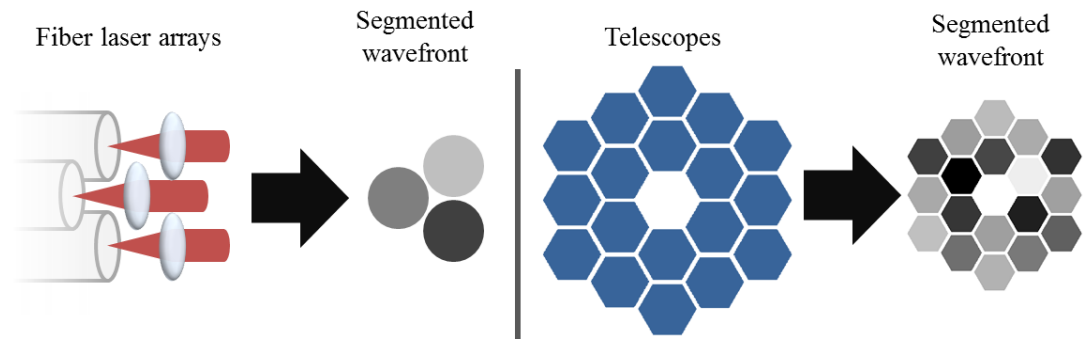

Figure 1. Representative scheme of the segmented wavefront generation by collimated fiber laser arrays and segmented telescope technologies. Piston phase defaults are represented as one of the main research fields in the domain.

Further author information:

E-mail: bastien.rouze@onera.fr 
The PISTIL interferometer ${ }^{16,17}$ is a Piston and Tip/Tilt segmented wavefront analyzer developed to meet the metrology need on the new coherent combination laser benches and possibly others segmented surfaces. Its development, initiated recently, has led to promising results in terms of the measurement accuracy, repeatability and resolution. However, it remains to be demonstrated that it can easily interface to a system with a high dynamic range of phase defaults. We propose to carry out an experimental demonstration of this extension with PISTIL. In section 2, we will develop the concepts associated with our interferometric system and how we will extend the phase piston range. In section 3, we will present the experimental setup and a main result. Finally, we will briefly discuss on the future work based on this current validation of the dynamic range extension.

\section{PRINCIPLE OF TWO-WAVELENGTH INTERFEROMETRY APPLIED TO PISTIL}

\subsection{Principle of PISTIL interferometry}

The PISTIL interferometer belongs to the family of lateral shearing interferometry. ${ }^{18-21}$ Its working principle $^{16}$ (depicted in Figure 2) is based on two-wave interference of wavefront replicas propagating from two neighboring segmented elements. A collimated beams collection from the segmented surface enters the system passing through the hole mask. It results a wavefront selection of the central part of each of them. Then, they propagate to a diffractive element, whose geometry may vary with the input segmented surface. In case of a hexagonal mesh (the most common in coherent combination and telescopes), the grating produces 6 replicas of each subwavefront (3 pairs of \pm 1 ) in three directions $\left(0^{\circ},+120^{\circ},-120^{\circ}\right)$. The replicas thus propagate onto an optimal position where each pair from neighboring beams superimpose (one element in the hexagonal mesh is surrounded by a maximum of six others) and interfere as the hole mask created the appropriate spacing between each Zero-orders.

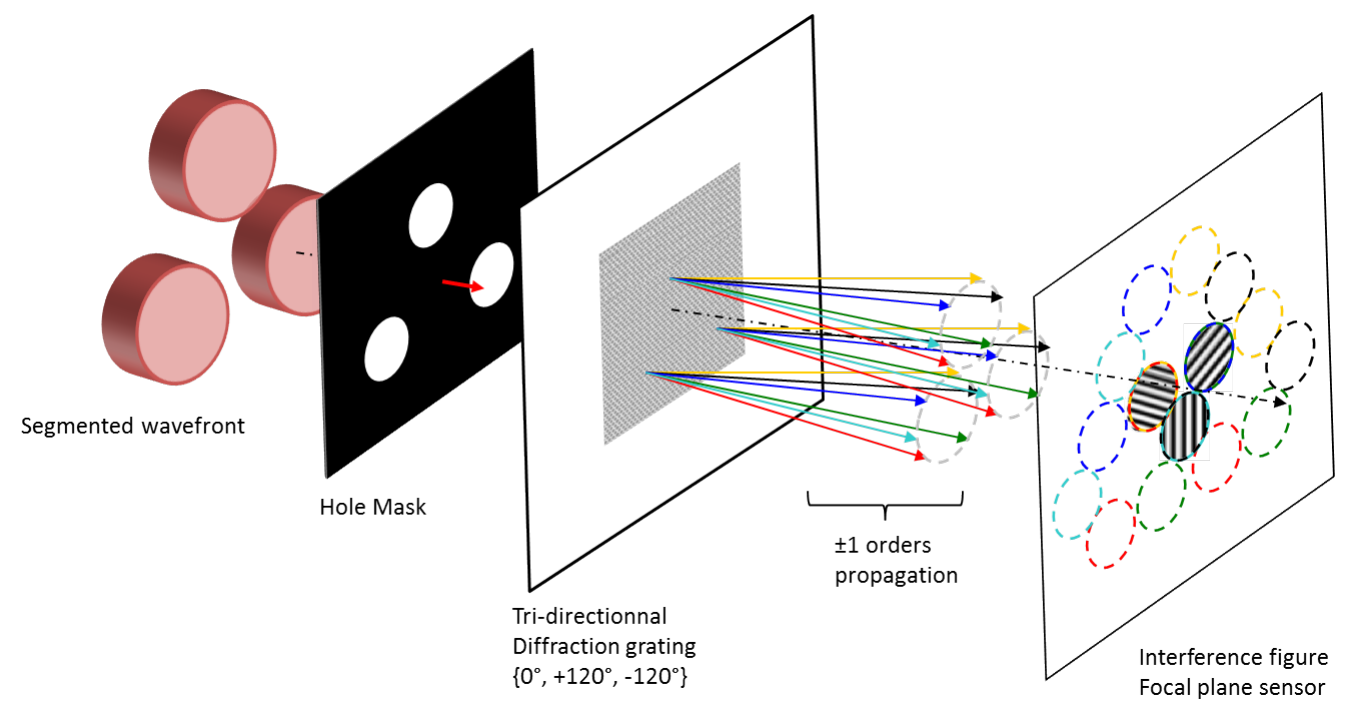

Figure 2. Layout of the interferometric setup. In this case, 3 collimated beams from a hexagonal mesh of a coherently combined laser array propagate through the PISTIL interferometer and generate the associated interference figure, called 'pistilogram'.

The fringes patterns define an interference figure called pistilogram. They are directly related to the combination of the subwavefronts of each fiber. We suppose that these subwavefronts can only have a combination of piston, tip and tilt. The fringes pattern gives clues about each relative phase difference (Figure 3) and we have the following description: 
Fringes pattern
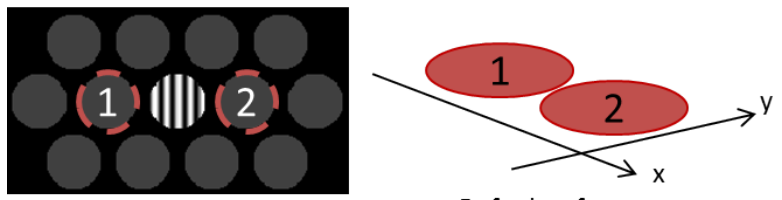

Default reference
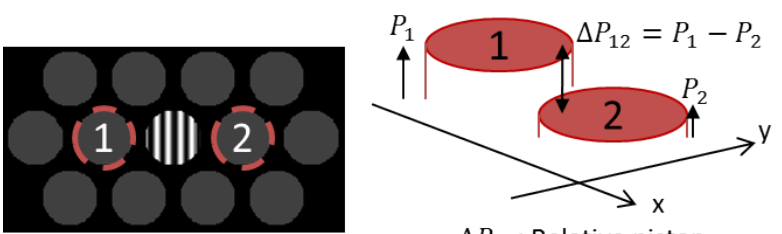

$\Delta P_{12}$ : Relative piston
Fringes pattern
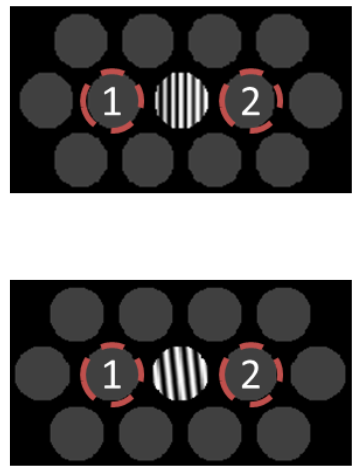

Relative phase
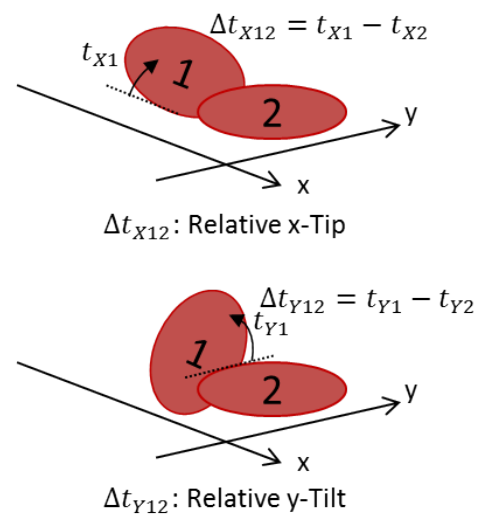

Figure 3. The behavior of the fringes is directly related to the relative wavefront default that exists between the two fibers. These defaults are measured relatively to a reference flat fringes pattern, in top left.

- $\Delta P_{12}=P_{1}-P_{2}$ is the piston difference (in optical path sense) between each subwavefront 1 and 2 (having absolute pistons $P_{1}$ and $P_{2}$ ). This optical path difference shifts the fringes, relatively to a pattern we call reference position (often $\Delta P=0$ ).

- $\Delta t_{X_{12}}=t_{X_{1}}-t_{X_{2}}$ is a tip difference (radian) between each subwavefront. It translates into a fringe frequency change along $x$.

- $\Delta t_{Y_{12}}=t_{Y_{1}}-t_{Y_{2}}$ is a tilt difference (radian) between each subwavefront. It translates into a fringe frequency change along $y$ (rotating fringes along the 1-2 axis in the Figure 3).

All these defaults can be estimated by the PISTIL technique. However, in the following, we will suppose that no tip/tilt occur during the segmented wavefront sensing (thus $\Delta t_{X_{12}}=\Delta t_{Y_{12}}=0$ ), as we will only focus on the piston effect on a measurement issue.

A detector is placed to record the interference figure. When it is done, we apply a Fourier Transform-based algorithm $^{17,22}$ which uses the reference pistilogram to derive the relative phase map. In case of piston distribution only, we derive thus $\widehat{\Delta P}_{12}$, (where hat denotes estimator) from our previous example and ultimately both $\widehat{P}_{1}$ and $\widehat{P}_{2}$, the estimate of absolute pistons, using a least-square inversion matrix reconstruction algorithm. ${ }^{23}$ In practice, the precision of the measure can reach a standard-error below $\lambda / 200$ in case of an optimal optical alignment. ${ }^{17}$

Calibration of the PISTIL interferometer is achieved by recording the pistilogram of a flat wavefront, which set all fringes pattern to a zero-point. It is the reference pistilogram. Then, there is no need to use, in practice, an external reference arm with a flat wavefront, making the system self-referenced.

\subsection{Extending the dynamic range with a Two-Wavelength setup (TWIPISTIL)}

For high pistons segmented wavefront metrology, measurements have to be conducted at any time, possibly in any configuration. The relative piston $\Delta P$ can only be known in a $2 \pi$-sized interval (in phase sense), as the fringes are periodic. In optical path sense, this $2 \pi$-wrapping is either a $\lambda$-wrapping in transmission wavefront analysis or $\lambda / 2$-wrapping in reflective wavefront analysis. In the following, we suppose a $\lambda$-wrapping, thus wrapping the relative piston within $[-\lambda / 2,+\lambda / 2[$, called the main determination interval.

If a temporal tracking of the phase variation can be recorded, unwrap the phase based on its continuous behavior is possible. But in a general case, or at the beginning of the temporal tracking, it is not possible to unwrap the phase this way. Then, Two-Wavelength Interferometry ${ }^{24-27}$ can be used to bypass this ambiguity, by generating a new virtual wavelength $\lambda_{v} \gg \lambda$ defined as

$$
\lambda_{v}=\lambda \cdot(\lambda+\Delta \lambda) /|\Delta \lambda| .
$$


where $\lambda+\Delta \lambda$ represents the second wavelength, and $\Delta \lambda$ the spectral gap. The main determination interval is then extended to $\left[-\lambda_{v} / 2,+\lambda_{v} / 2[\right.$.

To begin with, let say the relative piston $\Delta P$ is always known with a modulo $\lambda$. If we say $n \in \mathbb{Z}$ the modulo number, then we write

$$
\Delta P=\widehat{\Delta P}+n \lambda
$$

where $\widehat{\Delta P}$ is the raw estimate given by the processing algorithm. The use of two wavelengths $\lambda_{1}$ and $\lambda_{2}$ leads to two relative piston estimates $\widehat{\Delta P}^{\lambda_{1}}$ and $\widehat{\Delta P}^{\lambda_{2}}$ that are supposedly wrapped into $\left[-\lambda_{i} / 2 ; \lambda_{i} / 2\right.$ [ for $i=1,2$. We can write

$$
\left\{\begin{array}{l}
\Delta P=\widehat{\Delta P}^{\lambda_{1}}+n \lambda_{1}, \quad n \in \mathbb{Z} \quad(a) \\
\Delta P=\widehat{\Delta P}^{\lambda_{2}}+m \lambda_{2}, \quad m \in \mathbb{Z} \quad(b)
\end{array}\right.
$$

with $n$ and $m$ modulo numbers. We will use these estimates to find the couple $(n, m)$ that leads to the true estimate $\widehat{\Delta P}$ of the input relative piston. We represented the global idea in the scheme of Figure 4: we use a Vernier Effect with the second wavelength to validate which demodulation of $\widehat{\Delta P}^{\lambda_{1}}$ is the true estimate.

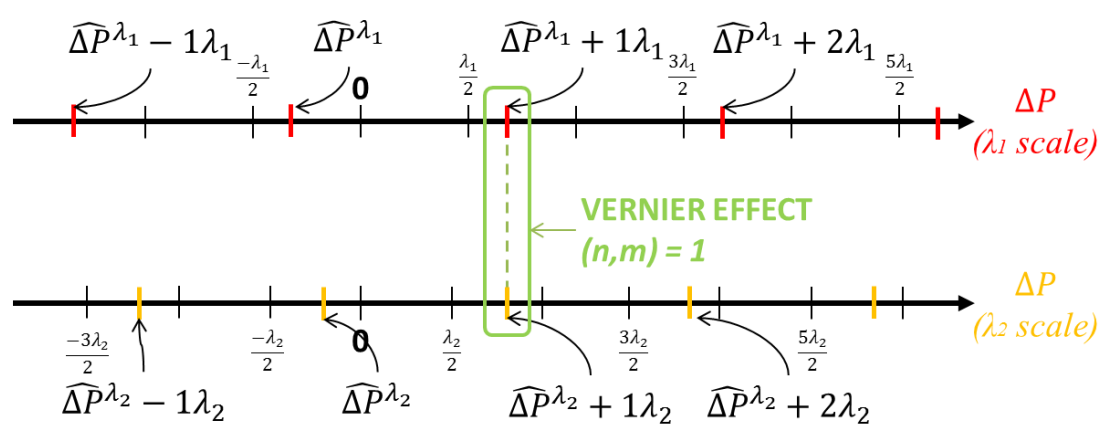

Figure 4. The two estimates $\widehat{\Delta P}$ are used to find the perfect match with the modulo numbers. The second wavelength is thus used to confirm the true value, as a caliper for a mechanical measurement.

In practice, we write $\lambda_{2}=\lambda_{1}+\Delta \lambda$, with $\Delta \lambda$ a spectral gap between the wavelengths. We can then define the virtual wavelength $\lambda_{v}$ as written in Eq. 1. As the Vernier effect is equivalent to the virtual wavelength measurement, with a main determination interval $\left[-\lambda_{v} / 2,+\lambda_{v} / 2\right.$ [, it bounds the $(n, m)$ solution couple to one possibility. Moreover, as $\lambda_{v}$ varies with $\Delta \lambda$, the smaller the spectral shift the bigger the extension.

Finally, the true estimate of the relative piston $\widehat{\Delta P}$ is reconstructed from both color measurements and we can compare their relative accuracy. We have accompanied our analysis with a proof of concept where 7 subwavefront elements were implemented. We set random pistons between $-0.5 \lambda$ and $2 \lambda$ and assumed two wavelengths of typical HeNe lasers (red at $633 \mathrm{~nm}$ and orange at $594 \mathrm{~nm}$ ). The results are shown in Figure 5 and are consistent: the second color, used for demodulate the relative pistons, allows to reconstruct the absolute piston map for both colors (one is represented); the residuals are similar for both colors, confirming estimation quality.
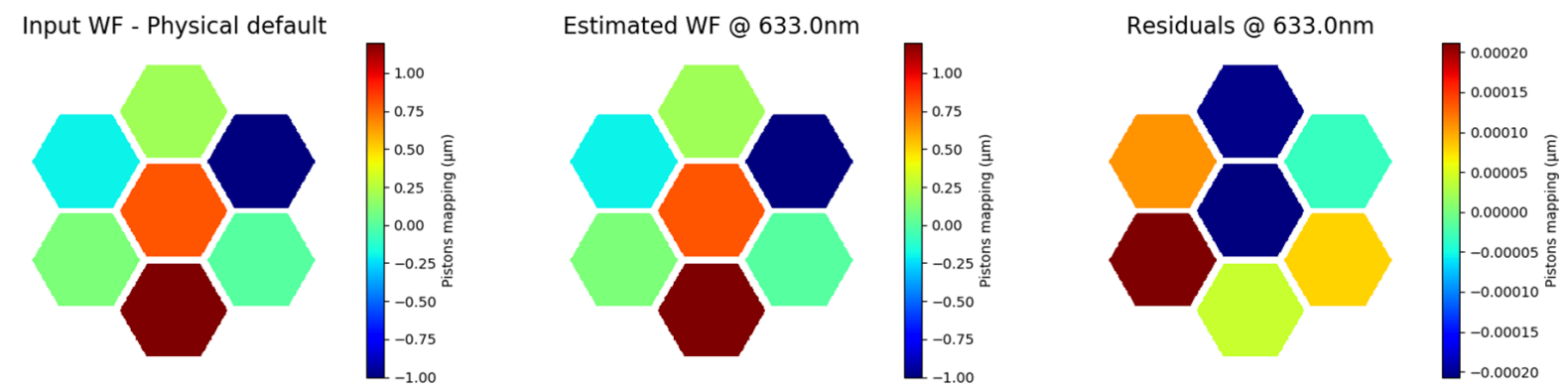

Figure 5. Simulation of random pistons reconstruction by TWI-PISTIL with two wavelengths (633nm and 594nm). 


\section{EXPERIMENTAL DEMONSTRATION OF TWIPISTIL}

\subsection{Experimental setup description}

A PISTIL interferometry testbed has been settled in our laboratory (Figure 6). We used a deformable mirror $(\mathrm{DM})$ as a segmented wavefront generator. The DM is an IRIS-AO PTT111 with 37 hexagonal segments of $700 \mu \mathrm{m}$ size arranged in a hexagonal mesh (3 rings of segments). Each segment has a stroke of $1.5 \mu \mathrm{m}$ in piston, and can also be tilted. Full description of the device and operation in piston and tilts modes are shown by Zamkotsian et al. ${ }^{28}$ For practical reasons, only two rings have been used for our work (a total of 19 segments). As the DM is a reflective device, a piston sets on a segment will have a double impact on the wavefront, therefore on the pistilogram $(0.4 \mu \mathrm{m}$ sensed as $0.8 \mu \mathrm{m})$. We will express the pistons in their doubled values to keep continuity with Section 2 (equivalent to transmission wavefront analysis).

The TWI is made up of two HeNe lasers with $\lambda_{1}=633 \mathrm{~nm}$ (red) and $\lambda_{2}=594 \mathrm{~nm}$ (orange). It will create a virtual wavelength of $\lambda_{v} \approx 9.64 \mu \mathrm{m}$ which allows enough dynamic extension relatively to the segmented elements' stroke (equivalent main determination interval $[-4.82 \mu \mathrm{m},+4.82 \mu \mathrm{m}[$ ). The lasers are filtered and collimated toward the DM. The wavefront generator is then conjugated to the mask, which has the same geometry than the DM, with a hole radius of $R_{h}=150 \mu \mathrm{m}$. The diffractive element is an intensity grating and has a Bravais period of $p_{g}=97 \mu \mathrm{m}$. It is positionned at a distance $z_{o p t} \approx 4.6 \mathrm{~cm}$. To decrease propagation of high diffraction orders, ${ }^{17}$ we added a pinhole between the grating and the focal plane array, in the Fourier plane.

The camera is a JAI-SP-5000-CXP4 visible+NIR CMOS with $2560 \times 2048$ px resolution (with $5 \mu \mathrm{m}$ pitch), and standard parameters (20 fps, 40ms exposure time) were used once the bench has been aligned and calibrated.

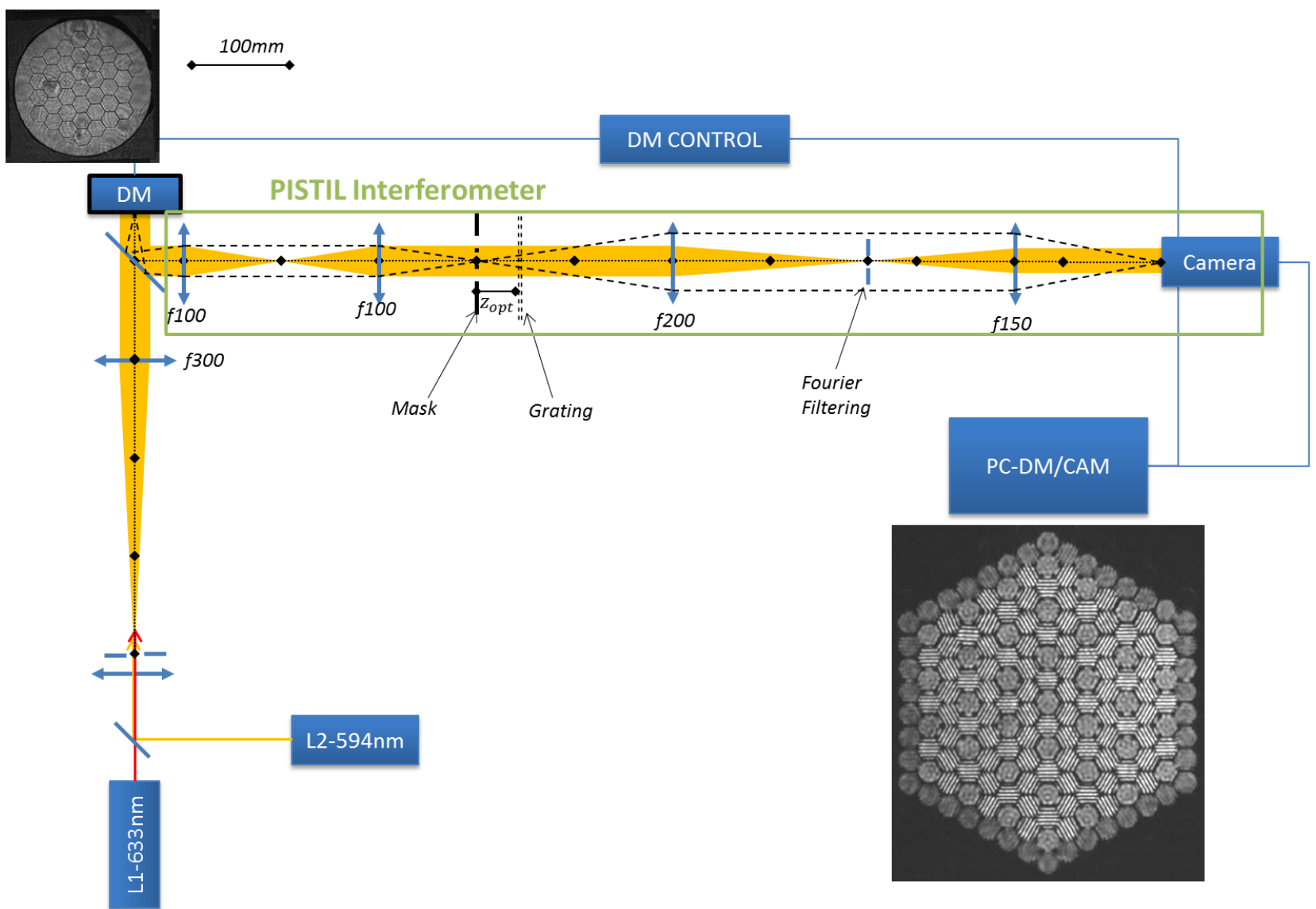

Figure 6. Scheme of the PISTIL testbed at ONERA, along with the two HeNe lasers. The DM plays the role of the segmented wavefront generator and is placed in a pupil plane. 


\subsection{Results}

First of all, for each color measurement, a flat reference wavefront is set on the DM, and the generated pistilogram is recorded by the camera. For each segmented wavefront sets on the DM, a 100 frames cubes is recorded. Each pistilogram is then computed by the Fourier-transform algorithm ${ }^{17}$ to derive the relative phase difference map. The two color relative phase maps are compared as explained in section 2 to determine for each the associated demodulation. The least-square reconstruction is achieved to obtain the absolute piston map. Global tip/tilt is removed by fitting a general tip/tilt over the segmented pistons map.

The color measurements have been done separately: we set 10 segmented wavefront maps with different piston values, some being above $\lambda_{1} / 2$ to trigger the necessity of the dynamic extension. We then switched lasers, moved the diffraction grating to the new optimal position (defined as $z_{o p t}=D \cdot p_{g} / 2 \lambda$, where $D$ in the distance between each segment), and did the same 10 measurements again. We present in Figure 7 along with Table 1 our results. Segments 8 to 19 are set to Zero-Point $(0 \mu \mathrm{m})$ allowing us to compare the residuals scales between both rings. Residuals for these segments are given by $\mathrm{min} / \mathrm{max}$ intervals for clarity reason.

\begin{tabular}{c|c|c|c} 
Segment number & Input $(\mu \mathbf{m})$ & Residuals @ $\lambda_{1}(\mathbf{n m})$ & Residuals @ $\lambda_{2}(\mathbf{n m})$ \\
\hline 1 & 0.8 & 1.0 & 4.2 \\
2 & 0.2 & 1.4 & 2.8 \\
3 & -1.0 & -5.3 & -4.0 \\
4 & 0.0 & -1.7 & -0.2 \\
5 & 1.2 & 5.4 & 1.9 \\
6 & 0.1 & 1.7 & 0.5 \\
7 & -0.2 & -1.1 & 1.1 \\
$8-19$ & $0.0-0.0$ & {$[-4.7,+3.0]$} & {$[-7.3,+4.3]$}
\end{tabular}

Table 1. List of the pistons composing the wavefront map of the presentation result. Segments 1,3 and 5 trigger the use of TWIPISTIL for correct estimation (absolute value greater than $\lambda_{1} / 2$ and $\lambda_{2} / 2$ ). Residuals are given in nanometers for best match with Figure 7 .

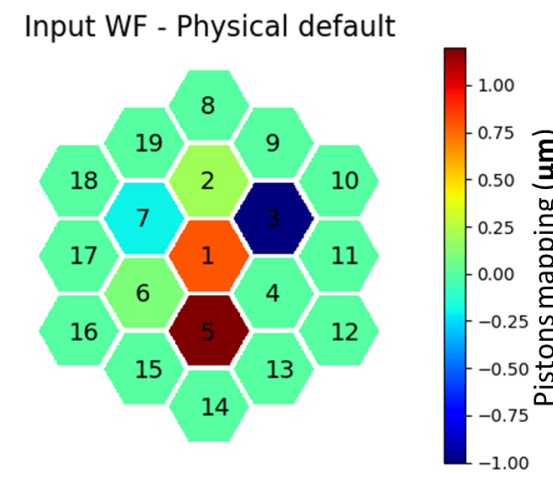

Estimated WF @ 633.0nm

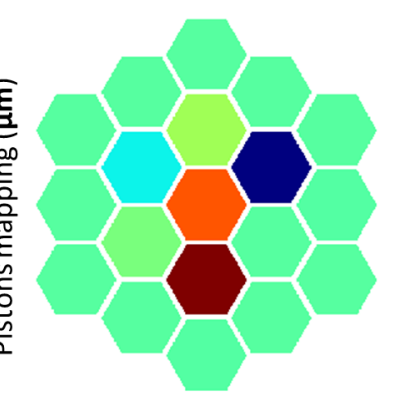

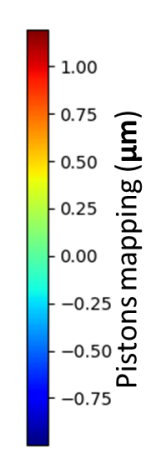

\section{Residuals @633.0nm}

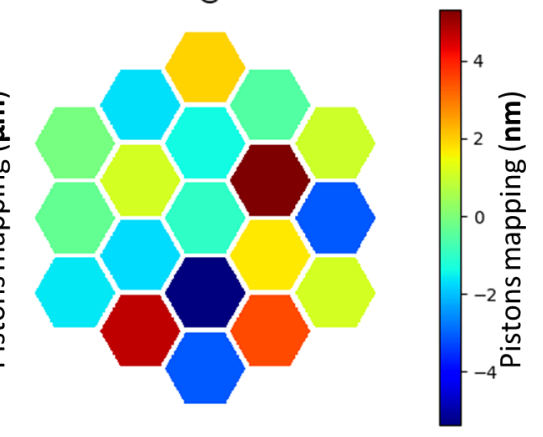

Figure 7. Experimental TWI-PISTIL measurement using the coefficients set of Table 1. The estimated absolute pistons map can be computed with both wavelengths. The residuals scale is set to nanometers to highlight the difference between the measure and the piston errors. One color is represented for clarity reason.

The result is in adequacy with our mathematical derivation and simulation. For this experiment, the standard error (standard deviation of the difference between measurements and input) is of $\lambda / 160$ while the peak-to-valley error is about $\lambda / 50$. The temporal noise in absolute piston (100-frames temporal standard deviation) is about $0.12 \mathrm{~nm}$ for the worst, which is consistent with the previous studies of segmented wavefronts using PISTIL.

Some source of errors can be identified in this work: the grating shift between each color measure could lead to precision loss along time. Also, a refocusing was needed for the collimation lense. These sources might be responsible for the quality loss of this TWIPISTIL setup, but can be fixed with high precision optics and automated positioning stages, especially for the grating. 


\section{CONCLUSION}

In order to provide an accurate wavefront metrology mean for the development of new segmented wavefrontbased systems, such as coherently combined high-power laser arrays, we worked on an open-loop probing issue: the high extension of the phase piston of each fiber laser. To be able to characterize it, we improved the PISTIL technique by doing two color measurements. We experimentally verified that the precision of TWIPISTIL is not much impacted by the separated color measurements (standard error below $\lambda / 150$ with a Peak-to-valley of $\lambda / 50)$. The interferometer remains self-referenced and needs only, two flat reference pistilograms - one for each

color- to be calibrated, without the need of an external arm.

The future work for the TWIPISTIL setup will focus on comparing its precision, raw noise and implementation process with a classical interferometric method; and reducing the spectral gap between $\Delta \lambda$. It will be necessary to use a specially designed ultra narrow bandpass filter to select, from a broad spectrum (tens of nm wide), two wavelengths for the process. The impact of the FWHM of the filter will be studied. Furthermore, the work will also focus on the development of a quasi-dynamic method to switch from one color to another. The proximity of the two wavelengths and the associated relative precision on wavelength selection (due to experimental conditions, mainly related to filter tilt precision) will be an opportunity to apply the Lofdahl and Eriksson algorithm. ${ }^{29}$ Ultimately, the technique will be applied to the open-loop metrology of a femtosecond high-power laser made up of coherently combined fiber lasers, for external noise sources probing.

\section{REFERENCES}

[1] Mourou, G. A., Hulin, D., and Galvanauskas, A., "The road to high peak power and high average power lasers: Coherentamplificationnetwork (can)," AIP Conference Proceedings 827(1), 152-163 (2006).

[2] Daniault, L., Bellanger, S., Le Dortz, J., Bourderionnet, J., Lallier, É., Larat, C., Antier-Murgey, M., Chanteloup, J.-C., Brignon, A., Simon-Boisson, C., and Mourou, G., "Xcan - a coherent amplification network of femtosecond fiber chirped-pulse amplifiers," The European Physical Journal Special Topics 224, 2609-2613 (Oct 2015).

[3] Yu, H. L., Zhang, Z. X., Wang, X. L., Su, R. T., Zhang, H. W., Ma, Y. X., Zhou, P., and Chen, J. B., "High average power coherent femtosecond pulse combining system based on an all fiber active control method," Laser Physics Letters 15, 075101 (may 2018).

[4] Müller, M., Klenke, A., Steinkopff, A., Stark, H., Tünnermann, A., and Limpert, J., "3.5kw coherently combined ultrafast fiber laser," Opt. Lett. 43, 6037-6040 (Dec 2018).

[5] Danson, C. N., Haefner, C., Bromage, J., Butcher, T., Chanteloup, J.-C. F., Chowdhury, E. A., Galvanauskas, A., Gizzi, L. A., Hein, J., Hillier, D. I., and et al., "Petawatt and exawatt class lasers worldwide," High Power Laser Science and Engineering 7, e54 (2019).

[6] Bellanger, C., Toulon, B., Primot, J., Lombard, L., Bourderionnet, J., and Brignon, A., "Collective phase measurement of an array of fiber lasers by quadriwave lateral shearing interferometry for coherent beam combining," Opt. Lett. 35, 3931-3933 (Dec 2010).

[7] Bourderionnet, J., Bellanger, C., Primot, J., and Brignon, A., "Collective coherent phase combining of 64 fibers," Opt. Express 19, 17053-17058 (Aug 2011).

[8] Dortz, J. L., Heilmann, A., Antier, M., Bourderionnet, J., Larat, C., Fsaifes, I., Daniault, L., Bellanger, S., Boisson, C. S., Chanteloup, J.-C., Lallier, E., and Brignon, A., "Highly scalable femtosecond coherent beam combining demonstrated with 19 fibers," Opt. Lett. 42, 1887-1890 (May 2017).

[9] Benna, B., Lombard, L., Jolivet, V., Delezoide, C., Pourtal, E., Bourdon, P., Canat, G., Vasseur, O., and Jaoun, Y., "Brightness scaling based on $1.55 \mathrm{~m}$ fiber amplifiers coherent combining," Fiber and Integrated Optics 27(5), 355-369 (2008).

[10] Geng, C., Luo, W., Tan, Y., Liu, H., Mu, J., and Li, X., "Experimental demonstration of using divergence cost-function in spgd algorithm for coherent beam combining with tip/tilt control," Opt. Express 21, 2504525055 (Oct 2013).

[11] "https://www.gmto.org/."

[12] "https://www.eso.org/sci/facilities/eelt/." 
[13] Chanan, G., Troy, M., Dekens, F., Michaels, S., Nelson, J., Mast, T., and Kirkman, D., "Phasing the mirror segments of the keck telescopes: the broadbandphasing algorithm," Appl. Opt. 37, 140-155 (Jan 1998).

[14] Esposito, S., Pinna, E., Puglisi, A., Tozzi, A., and Stefanini, P., "Pyramid sensor for segmented mirror alignment," Opt. Lett. 30, 2572-2574 (Oct 2005).

[15] Surdej, I., Yaitskova, N., and Gonte, F., "On-sky performance of the zernike phase contrast sensor for the phasing of segmented telescopes," Appl. Opt. 49, 4052-4062 (Jul 2010).

[16] Deprez, M., Bellanger, C., Lombard, L., Wattellier, B., and Primot, J., "Piston and tilt interferometry for segmented wavefront sensing," Opt. Lett. 41, 1078-1081 (Mar 2016).

[17] Deprez, M., Wattellier, B., Bellanger, C., Lombard, L., and Primot, J., "Phase measurement of a segmented wave front using piston and tilt interferometry (pistil)," Opt. Express 26, 5212-5224 (Mar 2018).

[18] Primot, J., "Three-wave lateral shearing interferometer," Appl. Opt. 32, 6242-6249 (Nov 1993).

[19] Primot, J. and Guérineau, N., "Extended hartmann test based on the pseudoguiding property of a hartmann mask completed by a phase chessboard," Appl. Opt. 39, 5715-5720 (Nov 2000).

[20] Velghe, S., Primot, J., Guérineau, N., Cohen, M., and Wattellier, B., "Wave-front reconstruction from multidirectional phase derivatives generated by multilateral shearing interferometers," Opt. Lett. 30, 245247 (Feb 2005).

[21] Mousset, S., Rouyer, C., Marre, G., Blanchot, N., Montant, S., and Wattellier, B., "Piston measurement by quadriwave lateral shearing interferometry," Opt. Lett. 31, 2634-2636 (Sep 2006).

[22] Freischlad, K. R. and Koliopoulos, C. L., "Modal estimation of a wave front from difference measurements using the discrete fourier transform," J. Opt. Soc. Am. A 3, 1852-1861 (Nov 1986).

[23] Penrose, R., "A generalized inverse for matrices," Mathematical Proceedings of the Cambridge Philosophical Society 51(3), 406413 (1955).

[24] Polhemus, C., "Two-wavelength interferometry," Appl. Opt. 12, 2071-2074 (Sep 1973).

[25] Velghe, S., Guérineau, N., Haïdar, R., Toulon, B., Demoustier, S., and Primot, J., "Two-color multi-wave lateral shearing interferometry for segmented wave-front measurements," Opt. Express 14, 9699-9708 (Oct 2006).

[26] Toulon, B., Primot, J., Gurineau, N., Hadar, R., Velghe, S., and Mercier, R., "Step-selective measurement by grating-based lateral shearing interferometry for segmented telescopes," Optics Communications 279(2), $240-243(2007)$.

[27] Houairi, K. and Cassaing, F., "Two-wavelength interferometry: extended range and accurate optical path difference analytical estimator," J. Opt. Soc. Am. A 26, 2503-2511 (Dec 2009).

[28] Zamkotsian, F., Lanzoni, P., Barette, R., Helmbrecht, M., Marchis, F., and Teichman, A., "Operation of a moems deformable mirror in cryo: Challenges and results," Micromachines 8(8) (2017).

[29] Lofdahl, M. G. and Eriksson, H., "Algorithm for resolving 2pi ambiguities in interferometric measurements by use of multiple wavelengths," Optical Engineering 40(6), $984-990$ (2001). 